\title{
JUSTIFICACIÓN DE LA ELIMINACIÓN DEL SARAMPIÓN
}

La Organización Mundial de la Salud dentro de sus objetivos de «Salud para todos en el siglo XXI» $y$, con respecto a las enfermedades transmisibles, en su objetivo $9 .^{\circ}$ se rcficre al control, eliminación o erradicación de algunas de ellas. Entre las diferentes tareas a desarrollar en los próximos años se encuentran las correspondientes a conseguir los objetivos: reducir la mortalidad y morbilidad del sarampión en la región Europea y eliminar el sarampión indígena en la región para el año 2007.

Ello implica la realización de diferentes tareas y el planteamiento de una serie de actividades que cualquier objetivo de control, eliminación o erradicación de estas enfermedades lleva consigo.

Éstas son:

A) Vigilancia de la enfermedad y estudio de brotes epidémicos, control de los mismos y estudio de la eficacia vacunal.
B) Control de susceptible que, junto con políticas de vacunación, nos lleven a la reducción de acumulación de los mismos.

C) Designación de laboratorios de diagnóstico y de referencia para el estudio de las cepas circulantes y su tipificación. A nivel nacional, se designará un laboratorio de referencia que esté en contacto con el europeo.

Por ello, la Dirección General de Salud Pública (Ministerio de Sanidad y Consumo) y el Centro Nacional de Epidemiología (Instituto de Salud Carlos III) han organizado una reunión científica en la que los diferentes temas que se tratan están en la relación con: Las estrategias de eliminación del sarampión en la Región Europea y a nivel mundial, programa de eliminación en las Américas, situación en España y abordaje del diagnóstico de la enfermedad en el laboratorio. 